\title{
Preliminary Evaluation of Plasmix Compound from Plastics Packaging Waste for Reuse in Bituminous Pavements
}

\author{
Clara Celauro ${ }^{1,2, *(\mathbb{D})}$, Rosalia Teresi ${ }^{2}$, Francesco Graziano ${ }^{1}$, Francesco Paolo La Mantia ${ }^{1,2} \mathbb{D}$ \\ and Antonio Protopapa ${ }^{3}$ \\ 1 Dipartimento di Ingegneria, Università degli Studi di Palermo, 90100 Palermo, Italy; \\ francesco.graziano@unipa.it (F.G.); francescopaolo.lamantia@unipa.it (F.P.L.M.) \\ 2 INSTM, Italian National Interuniversity Consortium of Materials Science and Technology, Via Giusti 9, \\ 50125 Florence, Italy; rosalia.teresi@unipa.it \\ 3 COREPLA, Italian National Consortium for the Collection and Recycling of Plastic packages, \\ 20100 Milan, Italy; protopapa@corepla.it \\ * Correspondence: clara.celauro@unipa.it; Tel.: +39-091-2389-9716
}

Citation: Celauro, C.; Teresi, R.; Graziano, F.; La Mantia, F.P.; Protopapa, A. Preliminary Evaluation of Plasmix Compound from Plastics Packaging Waste for Reuse in Bituminous Pavements. Sustainability 2021, 13, 2258. https://doi.org/ $10.3390 /$ su13042258

Academic Editor: Young-Ji Byon

Received: 30 December 2020

Accepted: 10 February 2021

Published: 19 February 2021

Publisher's Note: MDPI stays neutral with regard to jurisdictional claims in published maps and institutional affiliations.

Copyright: (c) 2021 by the authors. Licensee MDPI, Basel, Switzerland. This article is an open access article distributed under the terms and conditions of the Creative Commons Attribution (CC BY) license (https:/ / creativecommons.org/licenses/by/ $4.0 /)$.

\begin{abstract}
Finding an appropriate technical solution for reusing waste plastics is crucial for creating a circular plastic economy. Although mechanical recycling is the best option for recycling postconsumer plastics, some heterogeneous mixed plastics cannot be recycled to produce secondary material due to their very low properties. In this case, alternative routes should be considered in order to limit their disposal as much as possible. Therefore, in order to solve the environmental problems in the landfills of plastic waste recycling, and to improve the mechanical performance of bitumen for road pavement, the reuse of these post-consumer plastic wastes are preliminarily evaluated for the modification of bitumen for road use. The field of polymers used so far and widely studied concerns virgin materials, or highly homogeneous materials, in case of recycled plastics. In this work, a highly heterogeneous mixed plastic_Plasmix - from the separate collection in Italy, is used as a bitumen modifier for road construction. The research focused on the dry (into the mixture) and wet (into the binder) addition of different content of the Plasmix compound, with the aim of assessing the feasibility of the modification itself. Results of the mechanical tests carried out prove an increase in performance and that there is a potential of the addition of the Plasmix compound both for binder and mixture modifications.
\end{abstract}

Keywords: circular economy; sustainable reuse; plastic; waste; separate collection; polymer modified bitumen

\section{Introduction}

One of the environmental problems that afflicts not only Europe, but the whole world, is the increase in plastic waste daily produced. Nowadays, almost everything that surrounds us and is used in everyday life is made up of plastics of various types and nature, and increasing the recycling rate is becoming increasingly difficult, since waste flows from the recovery of plastic packaging have structures that are very heterogeneous and difficult to separate.

The recycling of post-consumer plastics from separate collection is a well-assessed process only if homogeneous polymers are reprocessed. Indeed, only in this case, the final properties of this secondary material are useful for several applications [1-3]. On the contrary, the recycling of heterogeneous polymers gives rise to secondary materials with low mechanical properties that can only be used for the production of items with ample thickness [4,5]. Moreover, the plastic products can undergo severe degradation processes during processing, and during the lifetime, that can dramatically reduce the properties of the materials [1-5]. Finally, during the separation of the various polymers in the industrial processes, it is not possible for the complete separation of all the polymers, 
and some mixed plastics cannot be mechanically recycled. This material usually goes to energy recovery systems.

Developing techniques for turning this waste into raw materials is indeed a global challenge for creating a circular economy for plastics. A possible use of this heterogeneous plastics mix could be as a modifier agent in bitumen. Considering the huge amount of bitumen and bituminous mixture yearly used for road construction and maintenance, a successful reuse of waste materials may promote the recycling of large quantities of waste that, otherwise, would be disposed in the landfills. In the last few years, in particular, this topic has gained increasing interest in both developed and developing countries [6-8]. Indeed, the use of plastic waste in asphalt pavements allows not only to recycle waste material, but also to obtain improvements in the performance of the mixtures intended for road construction [9].

In fact, in the last few decades, amongst the different possible materials, Polymer Modified Binders (PMB) determined significant improvements in the road pavement performance [6-8]. In particular, it is known in literature that modification of the bitumen with virgin thermoplastic polymers increases the bitumen stiffness and reduces its temperature susceptibility, particularly at high service temperatures [9-11]. For road application, two different methods are used, a so called "dry" and "wet" method [6,8,12].

The dry method incorporates the particles of plastic waste into hot aggregates before adding the binder, while in the wet method, the addition of the plastic occurs directly before mixing with the aggregate $[9,11,13]$. The main advantage of the dry process, compared to the wet method, is to require no or very limited processing for the re-use of the waste, and no modification of the traditional asphalt plant, for introducing the additive [14-16].

Therefore, it is particularly of interest to verify the feasibility of the modification with very heterogeneous waste plastic, considering the potential of this application as a possible solution for sustainable reuse of such a waste [17-19], as well as for potential reduction of the emission in construction of road pavement [20].

The aim of this work is to preliminarily investigate the feasibility of using the heterogeneous waste plastics as modifier in bitumen (wet method) or as additive in the mixture (dry method). For this purpose, particular attention was paid, in the case of the wet process, to the conventional properties required for a bituminous binder for road application (stability, viscosity and mixing temperature, mainly), in the case of the dry method, to the mechanical properties that are potentially improved by these waste plastics (resistance to permanent deformation, mainly).

\section{Materials and Methods}

\subsection{Materials Used}

\subsubsection{Bitumen}

The neat bitumen used in this research was a 50/70 penetration grade bitumen for road application: it was selected due to its large availability in Italy, and for assessing if the improvements produced by the modification can determine its practical adoption, at real scale.

The characteristics of the neat bitumen are summarized in Tables 1 and 2, in terms of mechanical properties and chemical fraction components, respectively. The chemical analysis, in detail, was carried out via thin layer chromatography and flame ionization detection (TLC/FID) technique.

Based on the results in Table 2, the Gaestel index (Ic) [21], i.e., the ratio of the total amount of asphaltenes and flocculants (saturates) to the total amount in peptizers (resins) and solvent, which is useful for denoting the colloidal stability of the bitumen, can be calculated for the bitumen used: $\mathrm{Ic}=(\mathrm{S}+\mathrm{As}) /(\mathrm{R}+\mathrm{Ar})=0.23$. Thus, considering the bitumen as a colloidal system, the optimal range for this index, for road use, is Ic $=0.22 / 0.5$ [22-25]. High values of the index denote the gel nature of the bitumen, as well as its low colloidal stability $[26,27]$. 
Table 1. Properties of neat bitumen.

\begin{tabular}{|c|c|c|c|}
\hline Characteristics & Standard & Unit & \\
\hline Penetration at $25^{\circ} \mathrm{C}$, pen & EN 1426 & $0.1 \cdot \mathrm{mm}$ & 67 \\
\hline Ring and ball softening point, $T_{R \& B}$ & EN 1427 & ${ }^{\circ} \mathrm{C}$ & 50.5 \\
\hline Penetration Index PI & EN 12591 & & -0.4 \\
\hline Ductility & EN 13589 & $\mathrm{~cm}$ & $>100$ \\
\hline $\begin{array}{l}\text { Penetration at } 25^{\circ} \mathrm{C} \text {, pen (after short term } \\
\text { ageing, according to EN 12607-1) }\end{array}$ & EN 1426 & $0.1 * \mathrm{~mm}$ & 64.7 \\
\hline $\begin{array}{l}\text { Ring and ball softening point, } T_{R \& B} \text { (after } \\
\text { RTOFT according to EN12607-1) }\end{array}$ & EN 1427 & ${ }^{\circ} \mathrm{C}$ & +3.5 \\
\hline Viscosity at $60^{\circ} \mathrm{C}$ & EN 13302 & $\mathrm{~Pa} \cdot \mathrm{s}$ & 255.5 \\
\hline Viscosity at $100{ }^{\circ} \mathrm{C}$ & EN 13302 & $\mathrm{~Pa} \cdot \mathrm{s}$ & 3.917 \\
\hline Viscosity at $135^{\circ} \mathrm{C}$ & EN 13302 & $\mathrm{~Pa} \cdot \mathrm{s}$ & 0.435 \\
\hline Viscosity at $150{ }^{\circ} \mathrm{C}$ & EN 13302 & $\mathrm{~Pa} \cdot \mathrm{s}$ & 0.222 \\
\hline
\end{tabular}

Table 2. Chemical components of neat bitumen (TLC/FID).

\begin{tabular}{ccccc}
\hline Component & Polarity & Percentage (\%) & Characteristics & Color \\
\hline $\mathrm{S}=$ Saturates & Non-polar & 2.4 & Viscous oil & White \\
Ar = Aromatics & Non-polar & 55.6 & Viscous liquid & Dark Brown \\
$\mathrm{R}=$ Resins & Highly polar & 25.4 & Solid to semi-solid & Dark Brown \\
As = Asphaltenes & Highly polar & 16.6 & Solid & Brown to black \\
\hline
\end{tabular}

\subsubsection{Plasmix}

The Plasmix additive [28] is made by the residual material of the sorting process of post-consumer plastics packaging waste, composed by different polymers and grinded in flakes to reduce shape and thickness. The Plasmix is characterized by a variable particle size, with average size about $6 \mathrm{~mm}$. At this stage, the Plasmix sample failed to mix with the bitumen: macroscopic phase separation between the dispersed polymer and the bitumen was detected in the blend. In fact, a series of preliminary tests showed that the crystalline fraction of PET contained in the Plasmix, which melts over $250{ }^{\circ} \mathrm{C}$, remained in significant quantities in the solid state without being dispersed in the bitumen. Based on the results of the mixing phase, the Plasmix was not used for producing the bitumen/additive blends to be tested.

Therefore, water flotation was performed on the Plasmix for density separation: the sample was washed and the heavy fraction (sunk in water) was separated from the light one (floating fraction). The light fraction, characterized by a density lower than that of the water at room temperature, consists of polyethylene (PE): (low-density polyethylene (LDPE), linear low-density polyethylene (LLDPE) and high-density polyethylene (HDPE), together with a small amount of polypropylene (PP). The heavy fraction mainly consists of polyethylene terephthalate (PET) with a lower polystyrene (PS) content and very small amounts of heterogeneous particles such as metals and paper. After water flotation, light to heavy fractions of the Plasmix were identified, in a ratio of about 20/80 in weight.

In order to better homogenize the Plasmix flakes in the molten state for the successive operations, the material was extruded using a twin screw extruder OMC (Officine Meccaniche Conte, Fondi, Latina, Italy). The extrusion was performed by setting a flat temperature profile at $270{ }^{\circ} \mathrm{C}$, the feed rate was set at $6 \mathrm{rpm}$ and the rotation speed of the screws was set at $120 \mathrm{rpm}$ (previous attempts at a temperature profile set at $100^{\circ} \mathrm{C}-120^{\circ} \mathrm{C}-$ $140{ }^{\circ} \mathrm{C}-160{ }^{\circ} \mathrm{C}-180{ }^{\circ} \mathrm{C}-200{ }^{\circ} \mathrm{C}-210{ }^{\circ} \mathrm{C}$, as typically set for extrusion of polyolefin, were carried out, too, but extrusion was not possible).

After extrusion at $270{ }^{\circ} \mathrm{C}$, the filament obtained were cut in a granulator; post granulation, the Plasmix pellets have a variable size, between 2 and $6 \mathrm{~mm}$. Figure 1 depicts the Plasmix samples before ("as is") and after extrusion. Figure 2 depicts the particle size distribution of the pellets, obtained via sieving. 


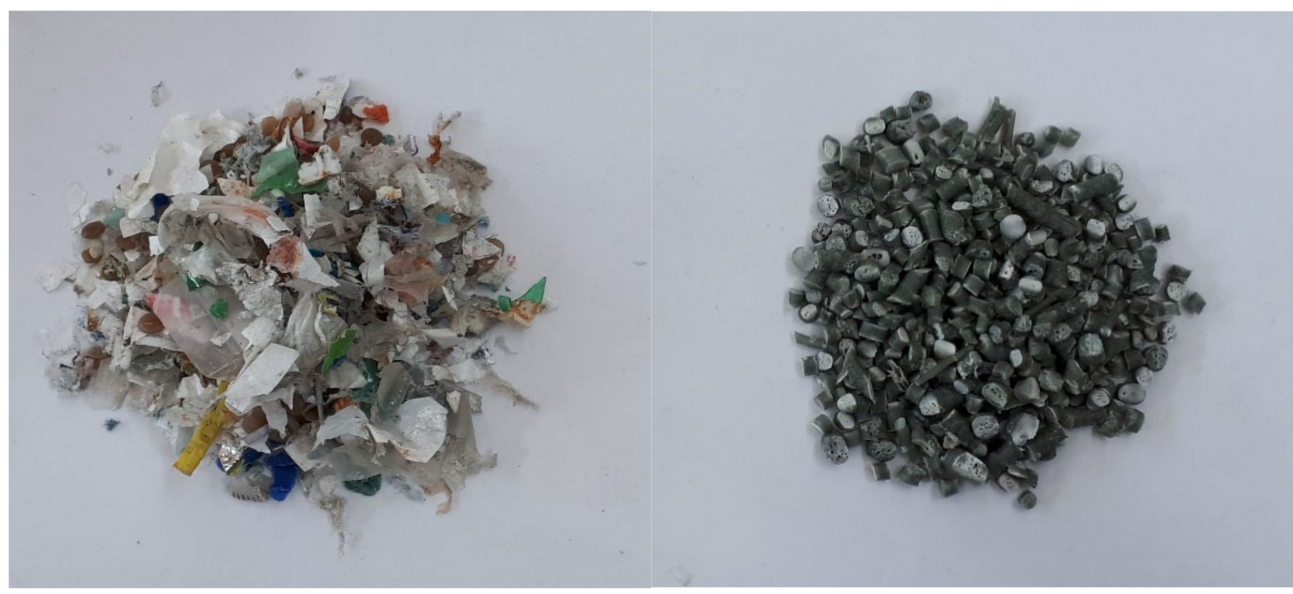

(a)

(b)

Figure 1. Plasmix sample: (a) Plasmix "as is"; (b) Pellets of Plasmix after extrusion and granulation.

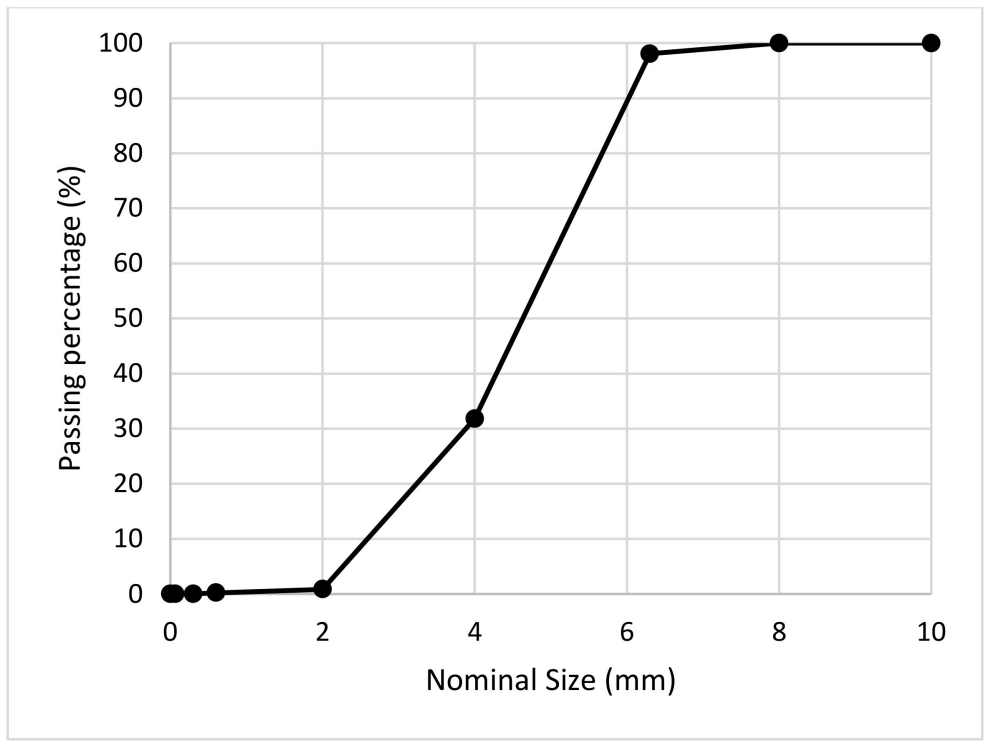

Figure 2. Particle size distribution of the extruded Plasmix.

Even after extrusion and granulation, the Plasmix sample failed to mix with the bitumen; again, severe problems of phase separation and evident inhomogeneity of the blend were experienced, due to the excess in heavy fraction in the Plasmix.

Therefore, recycled PE was added to the Plasmix, in order to increase the light to heavy fraction ratio to 50/50 (the same can be achieved by adding an adequate quantity of light fraction coming from the Plasmix itself). Another attempt of mixing with the bitumen was carried out, by introducing this mix of Plasmix in flakes and recycled PE (Plasmix "as is"), but it was not possible to produce a homogenous blend with the bitumen. Again, extrusion and granulation were performed, with the same processing described before, in order to improve homogeneity of the material and thus to facilitate the dispersion into the bitumen.

Finally, after the different attempts previously described-with this last polymer mixture-production of modified binders with the wet process was successfully performed. Polymer-modified bitumen samples were prepared at $180^{\circ} \mathrm{C}$ using a Silverson high shear mixer at a rotational speed of $3000 \mathrm{rpm}$ kept constant for $2 \mathrm{~h}$. Visual inspection during the mixing operations was continuously carried out in order to ensure the production of a smooth and fully homogeneous blend. The bitumen/Plasmix blends prepared in 
the laboratory for subsequent testing, and related production process, are summarized in Table 3.

Table 3. Bitumen/Plasmix blends prepared in the laboratory and production procedure.

\begin{tabular}{ccccc}
\hline Name & Plasmix (wt $\%)$ & Light/Heavy Fraction Ratio & Extrusion and Granulation & Mixing Result \\
\hline 2\%Plasmix & 2 & $20 / 80^{*}$ & NO & Fail \\
2\% Plasmix & 2 & $20 / 80^{*}$ & YES & Fail \\
$2 \%$ Plasmix “as is" & 2 & $50 / 50^{* *}$ & YES & Fail \\
2\% Plasmix 50/50 & 2 & $50 / 50^{* *}$ & YES & Success \\
$5 \%$ Plasmix 50/50 & 5 & $50 / 50^{* *}$ & YES & Success \\
\hline
\end{tabular}

* Original ratio in the Plasmix compound. ${ }^{* *}$ Ratio modified via introduction of recycled PE.

\subsubsection{Bituminous Mixtures and Aggregate}

The mineral aggregates used as skeleton for the mixtures to be tested came from a limestone quarry in Sicily: three different fractions and the filler have been composed in order to obtain the target mixture to be studied. The mix design obtained (mutual proportions of the component of the mixture, i.e., aggregate and bitumen) fulfills the requirements of the typical technical specification [29] for wearing courses for motorways.

Table 4 provides the physical and mechanical characteristics of the aggregates used, while Table 5 details the gradation of the different fractions of aggregate used. Considering the typical requirement for wearing courses in the Italian technical specifications also given in Table 4, the aggregate fractions selected are adequate for use in wearing courses in urban roads and rural roads belonging to the secondary network, with limited heavy vehicle traffic volume.

Table 4. Physical and mechanical characteristics of the aggregates.

\begin{tabular}{cccccccc}
\hline Characteristic & $\begin{array}{c}\text { Fraction } \\
\mathbf{1 0 - 1 5}\end{array}$ & $\begin{array}{c}\text { Fraction } \\
\mathbf{5 - 1 0}\end{array}$ & $\begin{array}{c}\text { Fraction } \\
\mathbf{0 - 6}\end{array}$ & Filler & Typical Requirement & Unit & Standard \\
\hline Density & 2.85 & $\mathbf{2 . 8 5}$ & $\mathbf{2 . 8 5}$ & & - & $\mathrm{Mg} / \mathrm{m}^{3}$ & $\mathrm{EN} \mathrm{1097-6}$ \\
\hline Fines contents & 2.6 & 2.7 & 8.8 & 1.2 & & EN 933-1 \\
\hline Sand Equivalent & - & - & 83 & & $\leq 15$ & EN 933-8 \\
\hline $\begin{array}{c}\text { Resistance to wear } \\
\text { (micro-Deval) }\end{array}$ & 11 & - & - & - & $\leq 20$ & EN 1097-1 \\
\hline $\begin{array}{c}\text { Los Angeles } \\
\text { Abrasion }\end{array}$ & 19.6 & 19.9 & - & - & - & EN 1097-2 \\
\hline
\end{tabular}

Table 5. Grading curve of the aggregate fractions used.

\begin{tabular}{ccccc}
\hline \multirow{2}{*}{ Sieve Opening [mm] } & Fraction 10-15 & Fraction 5-10 & Fraction 0-6 & Filler \\
\cline { 2 - 5 } & Passing\% & Passing \% & Passing \% & \% \\
\hline 25 & 100.00 & 100.00 & 100.00 & 100.00 \\
15 & 100.00 & 100.00 & 100.00 & 100.00 \\
10 & 62.69 & 99.82 & 100.00 & 100.00 \\
5 & 1.08 & 40.88 & 93.52 & 100.00 \\
2 & 1.06 & 1.11 & 62.03 & 100.00 \\
1 & 1.04 & 1.02 & 31.60 & 100.00 \\
0.425 & 1.03 & 0.91 & 5.92 & 100.00 \\
0.18 & 0.95 & 0.85 & 3.22 & 100.00 \\
0.075 & 0.86 & 0.76 & 2.14 & 81.90 \\
\hline
\end{tabular}

For the asphalt mixture to be produced with the dry process, the polymer additive was directly added to the hot aggregate (and not substituted to part of it) during the mixing process, prior to addiction of the binder. Considering the use as additive (since no part of 
the aggregate skeleton has been substituted), both the "as is" Plasmix (not-extruded) and the one after extrusion (Plasmix 50/50) were added in the mixture at a typical dosage for mixture's additives, i.e., the $2 \%$ in weight of aggregate, as better detailed in Section 3.3.

\subsection{Testing Methods}

\subsubsection{Chemical and Morphological Characterization of the Plasmix}

For evaluating the chemical structure of Plasmix, a Fourier transform infrared spectroscopy —attenuated total reflectance (FTIR-ATR) was performed, using a PerkinElmer's Spectrum Two, which detects the percentage absorbance as a function of the wavelength in thin films with a thickness of 100 microns in a range of $450-4000 \mathrm{~cm}^{-1}$. The instrument was equipped with a diamond (1 Reflection) ATR crystal. The spectrum was collected at room temperature, with a resolution of $4 \mathrm{~cm}^{-1}$.

The morphology of the pure Plasmix was observed using a Phenom Pro X Electron Scanning Microscope, SEM, (Phenom ProX, Phenom-World, Eindhoven, The Netherlands) and Energy-dispersive X-ray spectroscopy (EDX) spectroscopy analysis. Thermal characterization was carried out by differential scanning calorimetry DSC-60 Shimadzu (Shimadzu, Kyoto, Japan). The tests were performed in the presence of inert gas; the samples were subjected to heating/cooling/heating ramps in a temperature range between $40{ }^{\circ} \mathrm{C}$ and $290^{\circ} \mathrm{C}$, with a scanning speed of $20^{\circ} \mathrm{C} / \mathrm{min}$. Considering the high heterogeneity of the Plasmix, before testing, it was necessary to homogenize the material by extrusion.

\subsubsection{Characterization of the Blends Produced via "Wet Method"}

For the wet process, empirical penetration test and softening point were analyzed according to EN 1426 and EN 1427: Based on these results, the thermal susceptibility of the blend was evaluated, according to the Pfeiffer Penetration Index [30], as standardized in Annex A of the EN 12591:2009 Standard.

Modified blends were prepared with the addition of $2 \%$ and $5 \%$ of Plasmix and storage stability was evaluated in accordance with EN 13399, in an aluminum tube, placed for $72 \mathrm{~h}$ in an oven at $180{ }^{\circ} \mathrm{C}$. After storage at high temperature, the outer parts, upper and lower, were taken for penetration, softening point, and elastic recovery. Each characterization was performed in duplicate.

The morphology of the bitumen-Plasmix mixtures was investigated using an OPTIKA ${ }^{\circledR}$ (Ponteranica (BG), Italy) trinocular microscope, model N-400FL with a 4:1 magnification (lens 4/0.1 160/0.17). This method allowed to qualitatively describe the dispersion of polymers in modified binders [31] and the morphology results can usefully be related to the behavior of the modified mixtures [32].

The dynamic viscosity of the blend produced was also measured with a Brookfield viscometer (DV-III ${ }^{\mathrm{TM}}$ Ultra Rheometer, Middleboro, USA) on neat and modified binders, in order to determine the flow physical characteristics of the material and to guarantee that it can be pumped and handled at the hot mixing facilities; following EN 13302 standardized procedure, samples were tested at $100^{\circ} \mathrm{C}, 135^{\circ} \mathrm{C}, 150^{\circ} \mathrm{C}$ and $180^{\circ} \mathrm{C}$.

Dynamic mechanical analysis (DMA) of the modified binders studied was carried out using an Anton Paar Physica MCR 101 (Graz, Austria) dynamic shear rheometer (DSR), according to EN 14770 Standard. Frequency sweep tests in the range of linear viscoelastic behavior of the binders (applied strain $=1 \%$ ) were carried out from -10 to $180{ }^{\circ} \mathrm{C}$, with a plate-plate geometry, using the 8-mm plates or the 25-mm plates for low and high temperatures, respectively. The gap between the plates has been set equal to $1 \mathrm{~mm}$, in both cases. Isotherms of the complex shear modulus, $\mathrm{G}^{*}$, and phase angle, $\delta$, have been measured and those for the storage modulus, $G^{\prime}$, and loss modulus, $G^{\prime \prime}$, have been calculated from the tests.

Master curves of the viscoelastic functions were constructed assuming $\mathrm{T}=30^{\circ} \mathrm{C}$ as reference temperature, by horizontally shifting the experimental isotherms, based on the WLF (Williams-Landel-Ferry) shift factors [33]. 


\subsubsection{Characterization of the Mixtures Produced via "Dry Method"}

Mix design of the mixtures produced via dry method was carried out in the laboratory according to Marshall method. The optimal proportion of the components (aggregate fractions detailed in Tables 4 and 5 and base bitumen) is given in Table 6 .

Table 6. Optimal proportion of the components for the bituminous mixture studied.

\begin{tabular}{cc}
\hline Aggregate Fraction & Percentage \\
\hline Fraction 10/15 & $15 \%$ \\
Fraction 6/10 & $28 \%$ \\
Fraction 0/6 & $52 \%$ \\
Filler & $5.0 \%$ \\
Bitumen & $5.10 \%$ \\
\hline
\end{tabular}

Table 7 provides the final (target) gradation of the mixture composed according to the aggregate proportions given in Table 6, as well as the upper and lower acceptance limits as given in the technical specifications for the target mixture.

Table 7. Gradation of the mixtures produced and acceptance limits.

\begin{tabular}{cccc}
\hline \multirow{2}{*}{ Sieve $[\mathrm{mm}]$} & \multicolumn{2}{c}{ Acceptance Limits } & \multirow{2}{*}{ Final Mixture \% } \\
\cline { 2 - 3 } & Lower & Upper & \\
\hline 25 & 0.00 & 0.00 & 100.00 \\
15 & 100.00 & 100.00 & 100.00 \\
10 & 85.00 & 100.00 & 90.07 \\
5 & 30.00 & 45.00 & 39.80 \\
2 & 20.00 & 30.00 & 24.53 \\
0.425 & 10.00 & 20.00 & 13.47 \\
0.18 & 9.00 & 18.00 & 10.49 \\
0.075 & 8.00 & 13.00 & 9.29 \\
\hline
\end{tabular}

The mixture studied is therefore characterized by a continuously graded aggregate skeleton which ends up in a packed structure that, at the optimum binder content, results in a void content less than $5 \%$.

Since for this type of mixture, to be used in wearing courses, one of the most relevant properties is the resistance to permanent deformation at high service temperature; wheeltracking tests were conducted at laboratory scale, in order to simulate the conditions of the repeated load exerted to the pavement by the vehicular traffic, in accordance with EN 12697-22. Tests were carried out on bituminous concrete slabs having dimensions $305 \times 305 \times 50 \mathrm{~mm}$ at the air void content corresponding to the optimum from the mix design and equal to $\mathrm{v}=4 \%$. During the test, a solid rubber wheel applies a constant load of $700 \mathrm{~Pa}$ at the surface level of the mixtures, in a climatic chamber with $\mathrm{T}=60^{\circ} \mathrm{C}$, for a total of 20,000 passes; during each pass, the rut depth cumulated on the surface is measured.

\section{Results and Discussion}

\subsection{Plasmix Characterization}

Figure 3 shows a representative spectrum of a Plasmix pellet obtained by extrusion, obtained using FTIR-ATR technique (described in Section 2.2.1) was performed. Replicates carried out on different pellets proved a reasonable repeatability of the results. The analysis highlights two very intense bands, whose peaks, located respectively at $2916 \mathrm{~cm}^{-1}$ and $2849 \mathrm{~cm}^{-1}$, are to be referred to two stretching of the $\mathrm{CH}$ bonds that can be found in high-density polyethylene (HDPE), but also in other polyolefins and polyesters. 


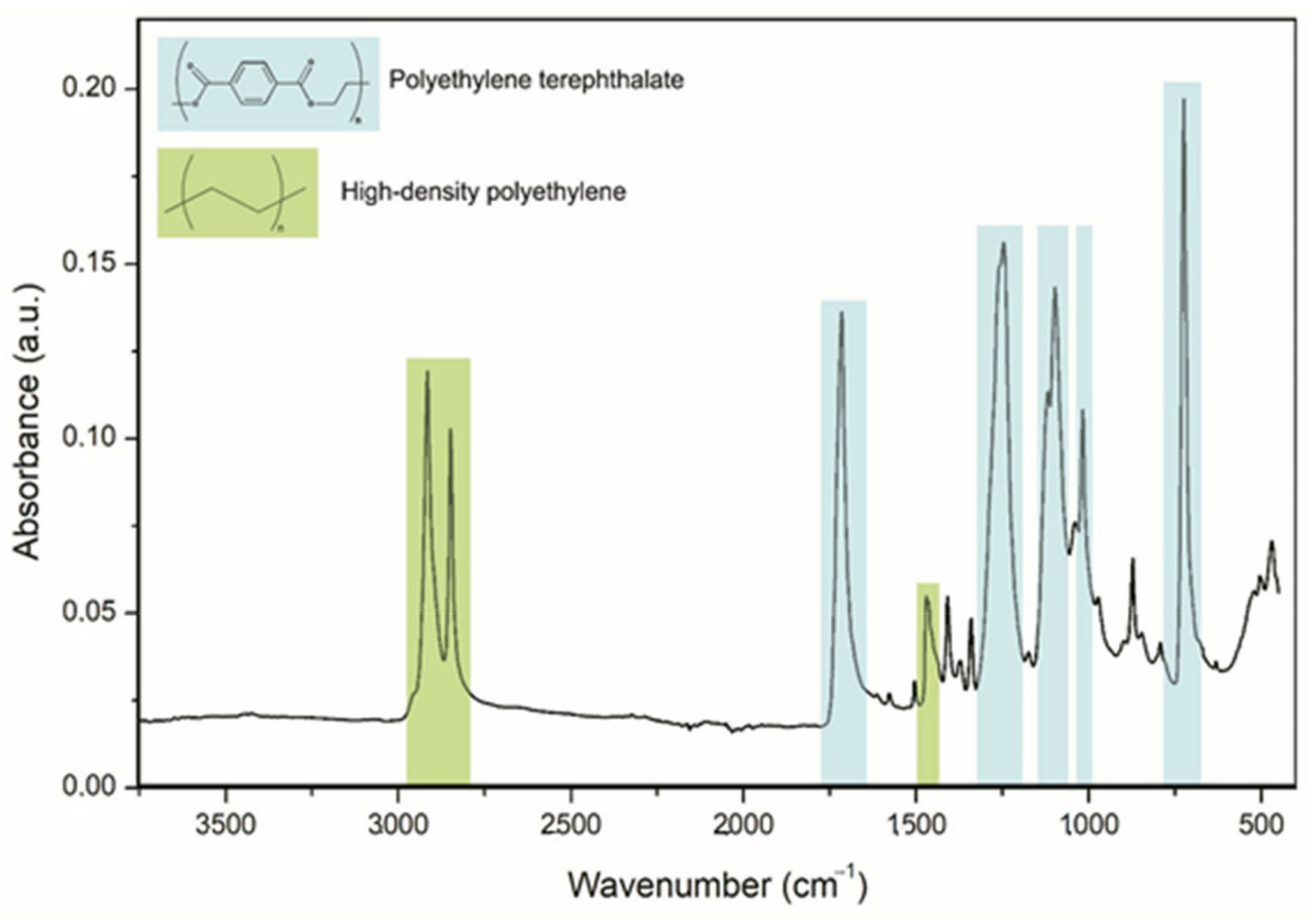

Figure 3. Spectrum of a Plasmix sample collected using FTIR-ATR.

Two other peaks can be observed at $1471 \mathrm{~cm}^{-1}$ and at $1465 \mathrm{~cm}^{-1}$, bending of the $\mathrm{CH}_{2}$ bond of HDPE. At $1725 \mathrm{~cm}^{-1}$, there is the peak that refers to the stretching of the $\mathrm{C}=\mathrm{O}$ bond present in the PET. In addition, the next two peaks at about $1247 \mathrm{~cm}^{-1}$ and at $1098 \mathrm{~cm}^{-1}$ for stretching the C-O ester bond of the PET. Finally, the last peak at $725 \mathrm{~cm}^{-1}$ is presumed to correspond to the out-of-plane bending of the $\mathrm{CH}$ bound to the benzene ring.

Figure 4 shows the SEM scan of the section of a Plasmix pellet. A substantial heterogeneity of the material clearly emerges from the enlargement, especially about the presence of non-polymeric particles.

In terms of components, the largest amount is detected for silicon ( $\mathrm{Si}$ ) and gold $(\mathrm{Au})$ with an atomic concentration of $41.04 \%$ and $40.63 \%$, respectively; also present, but less representative, there are aluminum $(\mathrm{Al})$ and ferrous components $(\mathrm{Fe})$, detected at $7.5 \%$ and $11.18 \%$, respectively, in terms of atomic percentage. It is reasonable to conclude that, amongst the components of Plasmix, some plastic material used for the construction of electronic circuits can be found.

DSC tests were carried on different samples of materials; again, test results proved a good repeatability. The results of the DSC test are shown in Figure 5. DSC tests were carried out on five different pellets of extruded Plasmix, having an average weight of about $8 \mathrm{mg}$. The testing protocol involved two heating ramps from 40 to $290{ }^{\circ} \mathrm{C}$ and a cooling ramp from 290 to $45{ }^{\circ} \mathrm{C}$ between them, at a constant heating and cooling rate of $20^{\circ} \mathrm{C} / \mathrm{min}$, for investigating the melting and crystallization behavior of the Plasmix. Thermograms obtained by the different replicates were reasonably reproducible. Figure 5 shows a representative result and it can be noticed that the heating cycle shows two main peaks, one around $115-130^{\circ} \mathrm{C}$ and one around $253^{\circ} \mathrm{C}$. A small peak around $166^{\circ} \mathrm{C}$ is also found. These melting temperature values confirm the presence of various polyethylene (LDPE, LLDPE and HDPE, PP and PET).

No DSC was carried out on the neat bitumen, based on the consideration that typical glass transition temperature $(\mathrm{Tg})$ for road-paving bitumen is in the range between $-15^{\circ} \mathrm{C}$ and $-30^{\circ} \mathrm{C}$ [27]. Therefore, such a very low $\mathrm{Tg}$ does not interfere with that of the dispersed polymers. 

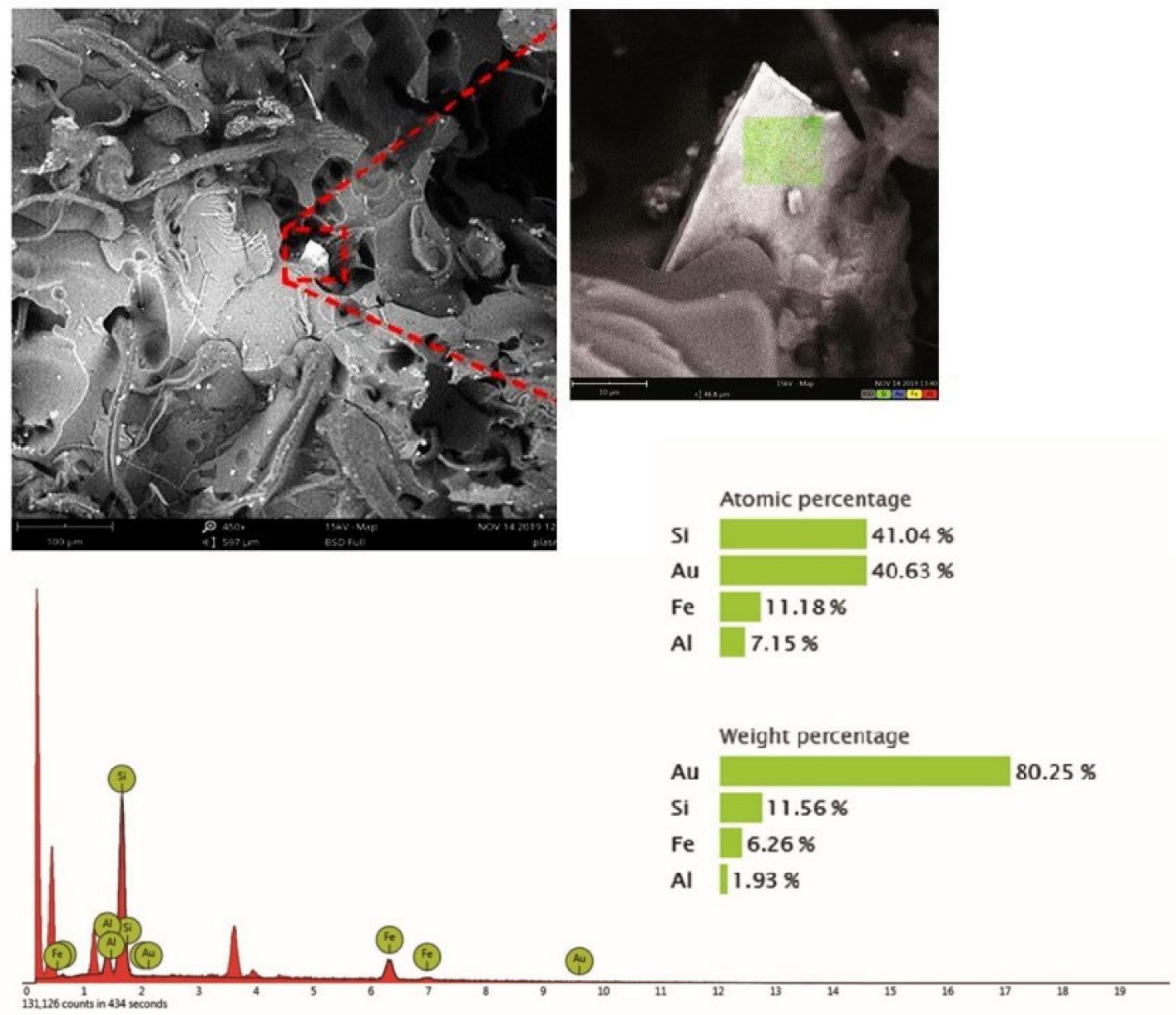

Figure 4. SEM and EDX analysis of a Plasmix sample.

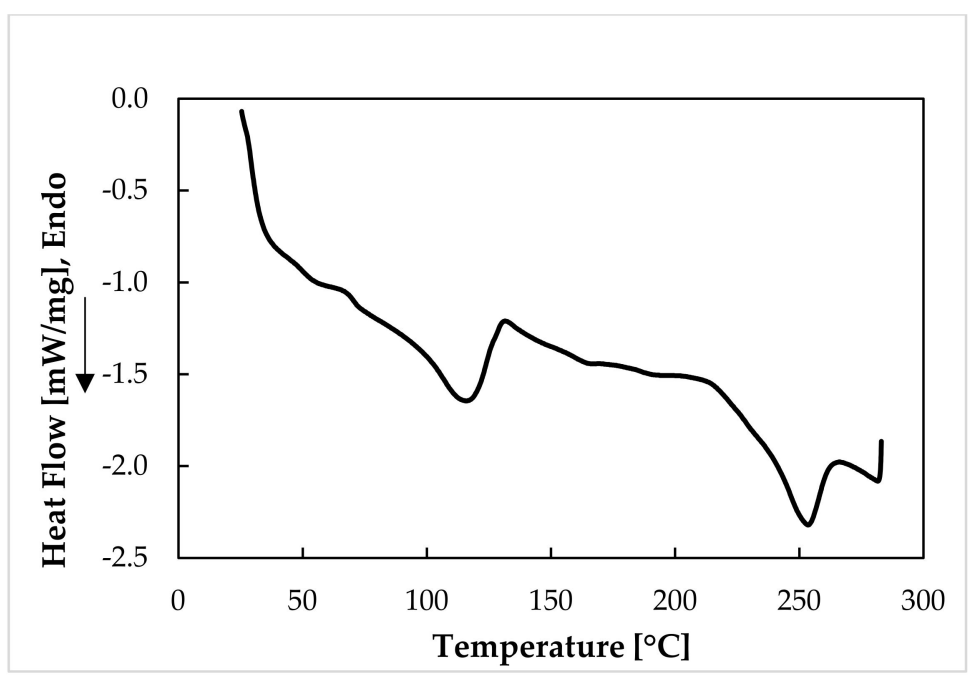

Figure 5. DSC heating scan $\left(+20^{\circ} \mathrm{C} / \mathrm{min}\right)$ of Plasmix.

\subsection{Bitumen and Modified Binders' Characterization \\ 3.2.1. Empirical Tests}

Empirical tests such as penetration grade and softening point were carried out on the modified binders produced via wet method and compared with those of the neat bitumen used as reference, for investigating the empirical properties typically considered in the technical specifications for road use. The results obtained are summarized in Table 8 . 
Table 8. Tests results for the neat bitumen and the binders modified with Plasmix.

\begin{tabular}{cccc}
\hline Requirement & Penetration at $\mathbf{2 5}{ }^{\circ} \mathbf{C}$ & Softening Point $\mathbf{T}_{\mathbf{R} \& \mathbf{B}}$ & Penetration Index, PI \\
\hline Standard & EN 1426 & EN 1427 & EN 12591 \\
Unit & $0.1 \cdot \mathbf{m m}$ & ${ }^{\circ} \mathrm{C}$ & \\
Neat bitumen 50/70 & 68 & 50.5 & -0.4 \\
$2 \%$ Plasmix 50/50 & 52.4 & 55 & 0.1 \\
$5 \%$ Plasmix 50/50 & 39.9 & 59 & 0.3 \\
\hline
\end{tabular}

As expected, the penetration at $25{ }^{\circ} \mathrm{C}$ decreased with the Plasmix addition, while the softening point increased. This is beneficial for the expected improvement at both moderate and high service temperature of the bituminous mixture produced with the modified binders, i.e., a higher resistance to repeated traffic loading and higher resistance to permanent deformation at high temperature. This improvement is also confirmed by the Penetration Index (PI) calculated according to Annex A to EN 12591 Standard, that can be considered a quantitative measure of the binders' thermal susceptibility [30]: the lower the Penetration Index, the higher the temperature sensitivity [23]. The typical requirement for binders for paving application is PI to range within -2 to +2 , being the higher values typical of PMBs. Comparing the results for the modified blend with the PI of the neat bitumen, the PI increases with the dosage of Plasmix 50/50 in the blend, suggesting a reduction in temperature susceptibility that will be better investigated via proper rheological characterization (see Section 3.2.3).

\subsubsection{Storage Stability Test}

After production via wet method, the modified binders underwent a storage stability test in order to monitor the phase separation between binder and polymers since it is considered a critical aspect of modification results. This test highlights the capacity of a bitumen to be stored inside the tanks at storage and distribution terminal, before transportation to asphalt production plants, and it guarantees that either storage or handlings do not cause significant physical or chemical alterations of the binder itself. The outer portions, top and bottom, were taken for penetration, softening point, elastic recovery and dynamic viscosity tests.

The EN 13399 Standard provides for a maximum variation between the softening point of the top and bottom equal to $3{ }^{\circ} \mathrm{C}$ and a variation of the penetration value not greater than $50.1 \cdot \mathrm{mm}$.

Storage stability is also the test that better indicates the compatibility between bitumen and polymer. In fact, if this is low, it is common for segregation effects to occur during the test during which the polymer tends to rise towards the top. This phenomenon determines a higher softening point at the top and a lower penetration index.

Table 9 details the storage stability results for the modified binders studied. Considering that storage stability requirement for PMBs is $\triangle \mathrm{TR} \& \mathrm{~B} \leq 5^{\circ} \mathrm{C}$, the results obtained for the bitumen modified with $5 \%$ of Plasmix 50/50 clearly indicate that these samples are to be considered as unstable: unreacted polymer was detected in bitumen and macroscopic phase separation occurred during its storage at high temperatures, which leads to undesirable problems during transportation of the binder and storage in the plant. On the other hand, the experimental results for both the samples modified at $2 \%$ of Plasmix 50/50 show a significant stability, adequate for road application.

A possible solution for the instability when using high content of Plasmix 50/50 may be limiting to the minimum the time between the production of the blend and its use in plant or site (this process is called "terminal blend production", in the case of modification of the bitumen with crumb rubber) [34] or studying the introduction of adequate compatibilizers for improving the stability of the blend and to avoid phase separation during storage at high temperature or in absence of stirring. In any case, for proper evaluation of the sustainability of the production of these blends, the overall emissions related to each process necessary for producing them should be taken into consideration, since, for example, the extrusion 
and granulation processes as well as the possible use of additive for stability purposes are likely to produce not-negligible impacts in terms of air pollutants and emissions [35].

Table 9. Storage stability results for the modified blends produced.

\begin{tabular}{|c|c|c|c|c|}
\hline Requirements & \multicolumn{4}{|c|}{ Storage Stability } \\
\hline Characteristic Standard & Pen @ $25{ }^{\circ} \mathrm{C}$ EN 1426 & $\Delta_{\text {Pen }}$ EN 13399 & $T_{R \& B}$ EN 1427 & $\Delta T_{R \& B} E N 13399$ \\
\hline Unit & $0.1 \cdot \mathrm{mm}$ & $0.1 \cdot \mathrm{mm}$ & ${ }^{\circ} \mathrm{C}$ & ${ }^{\circ} \mathrm{C}$ \\
\hline Bitumen 5\% Plasmix (50/50) top & 25 & \multirow{2}{*}{8.5} & $71-81$ & \multirow{2}{*}{ not applicable } \\
\hline Bitumen 5\% Plasmix $(50 / 50)$ bottom & 33.2 & & 64 & \\
\hline Bitumen $2 \%$ Plasmix $(50 / 50)$ top & 25 & \multirow{2}{*}{1.5} & 56.5 & \multirow{2}{*}{1} \\
\hline Bitumen $2 \%$ Plasmix $(50 / 50)$ bottom & 23.5 & & 55.5 & \\
\hline
\end{tabular}

\subsubsection{Morphology of the Modified Blends via Fluorescence Microscopy}

Morphology of the modified blends produced was studied via fluorescence microscopy and micrographs were taken on the blends immediately after production and after storage at high temperatures according to the procedure of the stability tests, in accordance with EN 13399. The micrographs obtained qualitatively show the dispersion of the polymer in the bituminous matrix [33]; at both dosages ( $2 \%$ and $5 \%)$, the dispersing phase remains the bitumen, while the Plasmix remains the dispersed phase.

In details, Figure 6 a proves that the $2 \%$ of Plasmix $50 / 50$ is a dosage too low and it does not significantly affect the base bitumen, as it will be better proved by results of the rheological characterization given in the following paragraph of Section 3.2.4. On the other hand, the dispersion of the $5 \%$ of Plasmix 50/50 is much more evident, with a homogenous distribution in the base bitumen, and this is also in line with the results of the rheological characterization, where substantial differences are detectable between the behavior of the base bitumen and that of the blend modified with the $5 \%$ of Plasmix 50/50, even though the phase inversion is not reached.

In terms of stability, after storage at high temperature, the blend with $2 \%$ of Plasmix $50 / 50$ shows no substantial difference in morphology between the top and bottom section of the sample, thus proving a substantial stability, in line with the results provided in Table 9. In the same way, the instability of the blend with $5 \%$ of Plasmix 50/50 can also be observed, since Figure $6 \mathrm{~d}, \mathrm{f}$-respectively taken on the top and bottom sections of the sample-very different from each other, thus proving the separation of the bitumen/plastic phases during the prolonged time at high temperature.

\subsubsection{Rheological Characterization}

Dynamic viscosity was determined for modified binders studied; the neat bitumen was also tested for comparison purposes. Figure 6 depicts the results of the viscosity tests carried out.

As can be seen from Figure 7, the viscosity of the plastic/bitumen blend increases with the content of Plasmix 50/50, as expected, in line with the results of the empirical tests, i.e., the increase in softening point and the decrease in penetration, given in Table 7 and previously discussed. This increase is beneficial for the resistance (stiffness) at high temperature, which is particularly of interest in regions with warm climate, such as those in the Mediterranean area. 


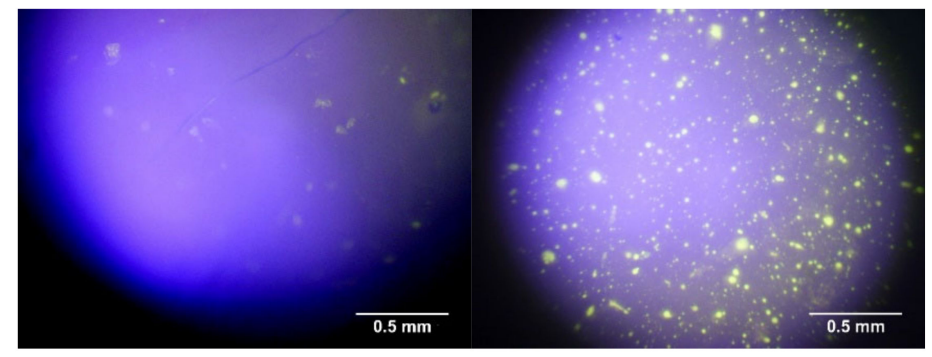

(a)

(b)

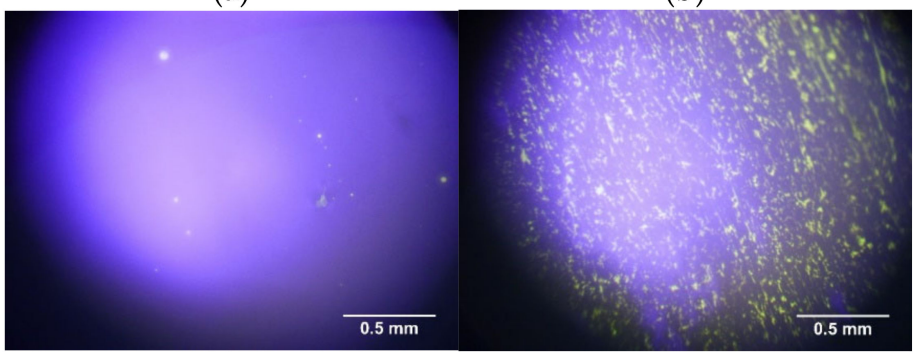

(c)

(d)

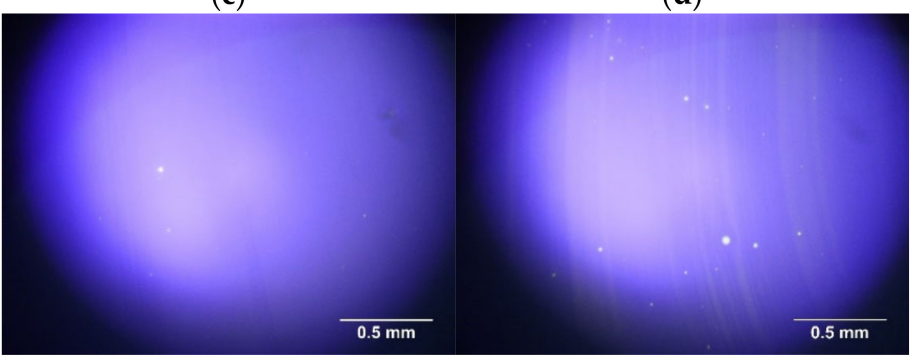

(e)

(f)

Figure 6. Micrographs of the plastic/bitumen blends after production and after storage at high temperature: (a) 2\% Plasmix 50/50; (b) 5\% Plasmix 50/50; (c) 2\% Plasmix 50/50 after storage-TOP; (d) 2\% Plasmix 50/50 after storage-BOTTOM; (e) 5\% Plasmix 50/50 after storage-TOP; (f) 5\% Plasmix 50/50 after storage-BOTTOM.

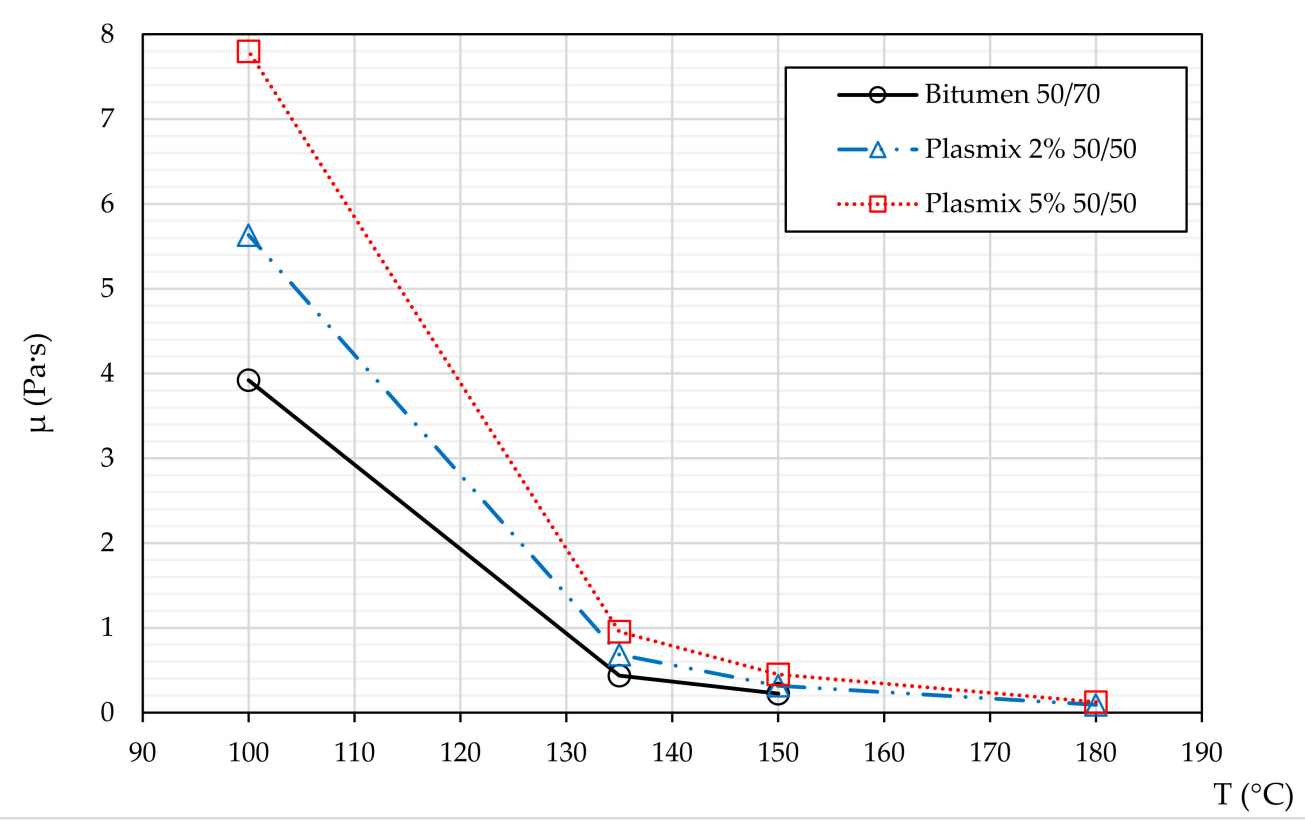

Figure 7. Dynamic viscosity of the binders studied, at the rotational viscometer Brookfield. 
In any case, this increase in viscosity does not compromise the possibility of using the modified binders produced for road applications. In fact, for each sample, the viscosity value at $135{ }^{\circ} \mathrm{C}$ and the equiviscosity temperature were evaluated, as detailed in Table 10. These viscosities are of interest at production level for bituminous mixtures, since the binder viscosity at $135^{\circ} \mathrm{C}$ is prescribed to be less than $3 \mathrm{~Pa} \cdot \mathrm{s}$ [36-38], for adequate pumpability from the storage tank to the mixing chamber in the plant. On the other hand, the equiviscosity temperature is the temperature at which the binder has a viscosity equal to $0.17 \mathrm{~Pa} \cdot \mathrm{s}$, that is considered as the ideal one for a complete and effective coating of the aggregates during the mixing phase at the asphalt plant $[36,37]$.

Table 10. Equiviscosity temperature and viscosity at $135^{\circ} \mathrm{C}$.

\begin{tabular}{ccc}
\hline Requirement & Equiviscosity T @ 0.17 Pa·s & Pumpability $\boldsymbol{\eta} @ \mathbf{1 3 5}{ }^{\circ} \mathbf{C}$ \\
\hline Unit & ${ }^{\circ} \mathbf{C}$ & Pa s \\
\hline Neat bitumen 50/70 & 153 & 0.432 \\
2\% Plasmix 50/50 & 159 & 0.682 \\
5\% Plasmix 50/50 & 169 & 0.95 \\
\hline
\end{tabular}

As said, the addition of Plasmix 50/50 increased the viscosity of the blends produced, but in any case, the requirement at $135{ }^{\circ} \mathrm{C}$ is fully satisfied, at both Plasmix dosages.

As far as the equiviscosity temperature is concerned, for the blend with addition of $5 \%$ of Plasmix 50/50, results prove an ideal mixing temperature about $15^{\circ} \mathrm{C}$ higher than that of the neat bitumen, while at $2 \%$ of Plasmix $50 / 50$, comparison with the neat bitumen used as reference, allows one to conclude that this dosage does not significantly affect the equiviscosity temperature (the difference between the results is lower than 5\%) and, therefore, the energy needed in the production process in asphalt plant.

The results obtained for the blend with $2 \%$ of Plasmix 50/50 prove that, compared with the one with 5\% Plasmix 50/50, the first is convenient from the technical, environmental and economical point of view; at lower mixing temperature there is lower risk of premature ageing of the binder as well as a lower need of energy for the production itself (lower costs and lower impacts).

The viscosity tests were also carried out on the samples, subject to storage stability procedure, i.e., taken on the top and bottom of the sections of the tubes used in accordance with EN 13399 Standard. Results are provided in Figure 8, where the viscosity of the modified blend is compared with that of the top and bottom sections taken after storage at high temperature. Two replicates were carried out for each test; due to the low repeatability for the blend with 5\% of Plasmix 50/50, the replicates could not be averaged, thus proving the high heteromogeneity of the samples. This means that the bitumen blend with $5 \%$ of Plasmix 50/50 is not stable enough to be safely subjected to the handling and transportation operations, from the production site to the asphalt plant. On the other hand, the results for the top and bottom section of the blend with $2 \%$ of Plasmix 50/50 after prolonged storage at high temperature, show high repeatability and are comparable with the viscosity of the modified blend before storage, thus proving its adequacy for road application. These results are fully in line with those of the storage stability evaluated via softening point and penetration variations, but better depict the rheological behavior and, thus, the risk of instability of the bitumen polymer blends.

The results of the DSR test performed are given in Figures 9 and 10, in terms of master curve of the storage modulus, $\mathrm{G}^{\prime}$, and Black diagram, respectively.

When analyzing the results obtained in terms of $G^{\prime}$ master curve (see Figure 9), one can conclude that after horizontal shifting at the reference temperature, using the shift factors according to Williams-Landel-Ferry (WLF) [31], all the isotherms merge into a smooth curve, so that Time-Temperature Superposition Principle (TTSP) seems to be respected by the blends tested. 

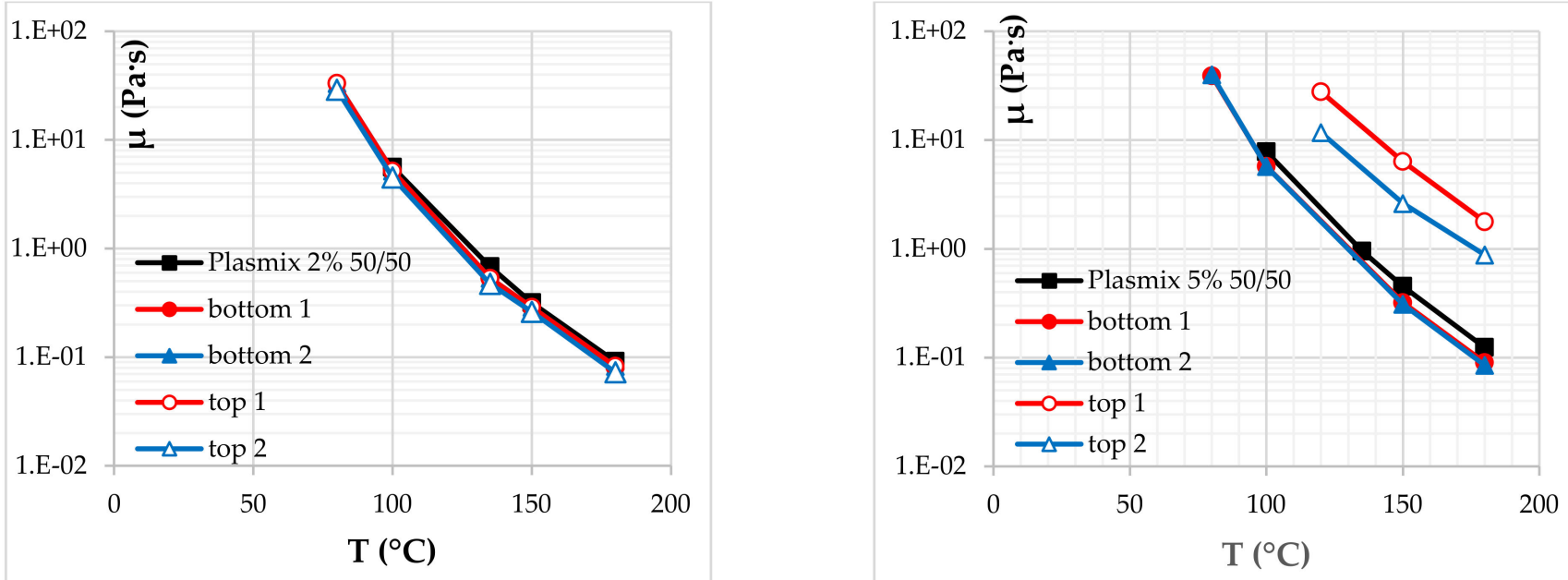

Figure 8. Master curves of the storage modulus, $\mathrm{G}^{\prime}$, at $30^{\circ} \mathrm{C}$, for top and bottom sections of all the binders studied.

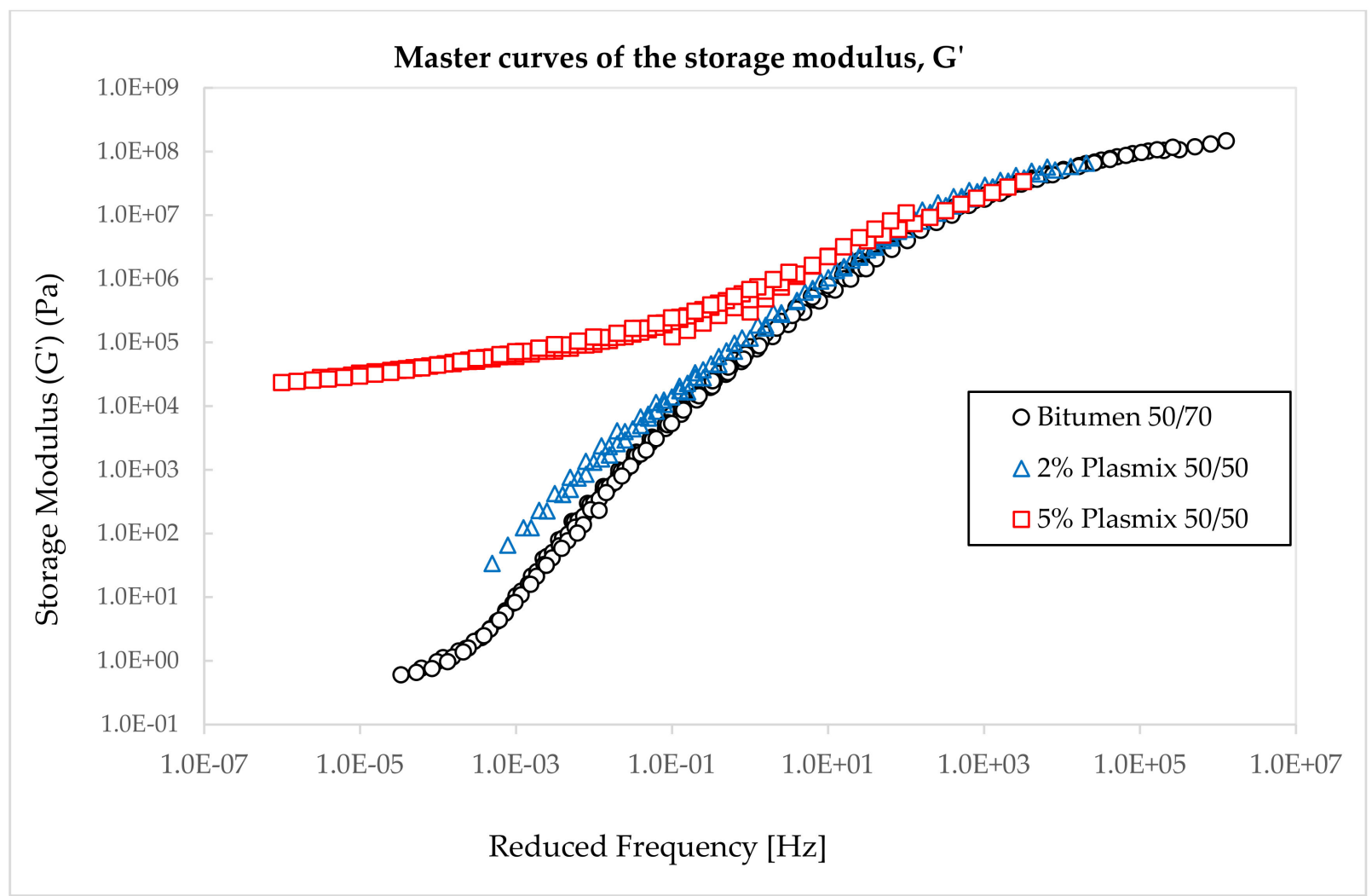

Figure 9. Master curves of the storage modulus, $\mathrm{G}^{\prime}$, at $30^{\circ} \mathrm{C}$, for all the binders studied. 


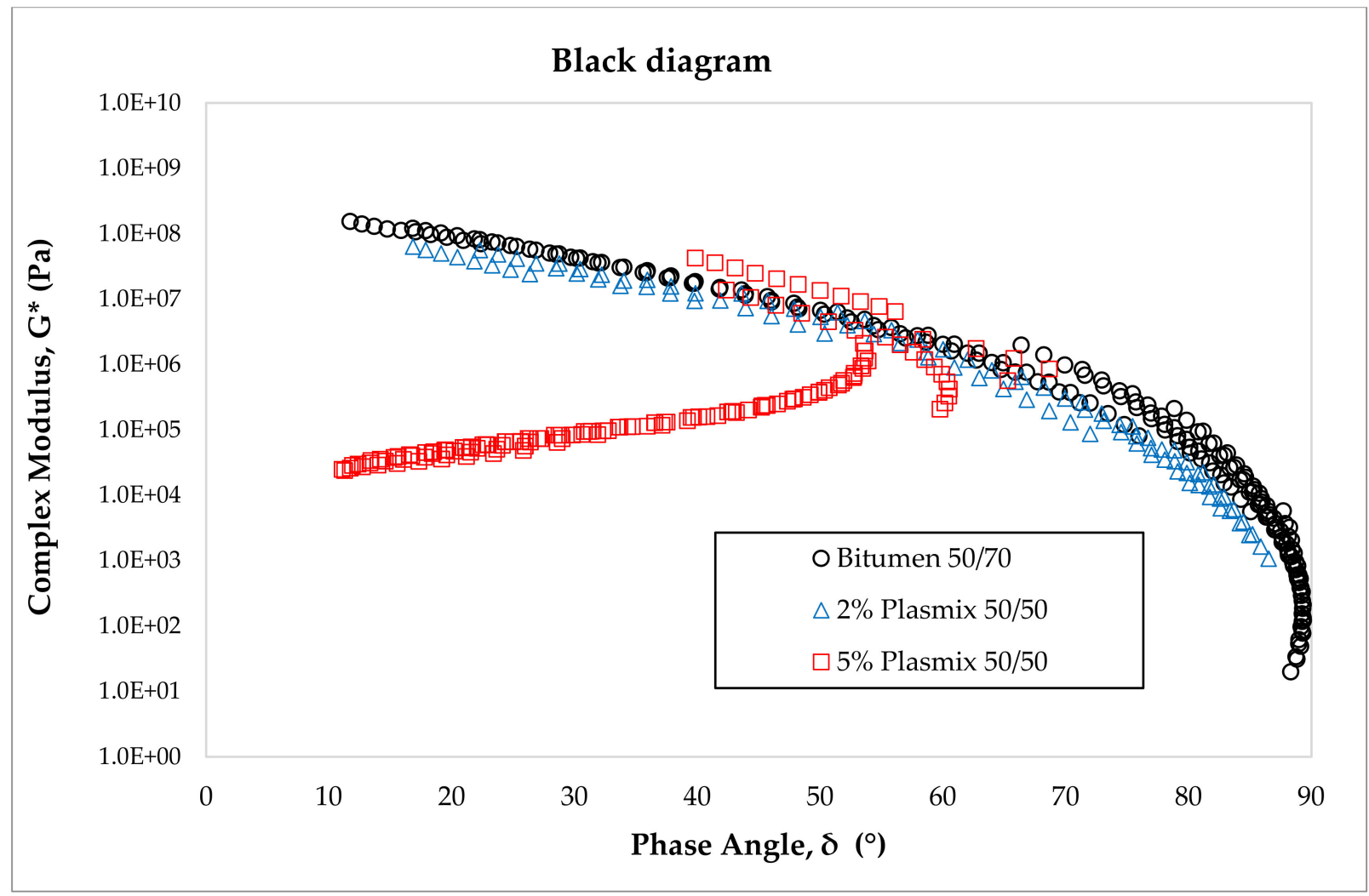

Figure 10. Black diagram for all the binders studied.

The bitumen modified with the $2 \%$ of Plasmix 50/50 does not exhibit a significant increase in elastic component, at the high testing frequencies (low temperature), but this increase became more appreciable at low frequencies (high temperature) [33]; this behavior is favorable, since the material is not too rigid at low temperature, but it proves a higher stiffness at low temperature (thus offering a higher resistance to the loads). The blend modified with the $5 \%$ of Plasmix 50/50 shows a much more elastic behavior than the blend modified with the $2 \%$, so that at very low frequencies-i.e., high service temperature-a plateau is reached, much higher than the corresponding one of the neat bitumen, that tends to zero.

The same results obtained during the frequency sweep tests at different temperatures can be directly plot in terms of Black diagrams (norm of $\mathrm{G}^{*} \mathrm{vs}$. phase angle), as given in Figure 10, with no need to invoke the TTSP. In this graph, the effect of Plasmix content is clear: at $2 \%$ of Plasmix 50/50, the behavior is still very similar to that of the neat binder, even if slightly shifted toward lower values of the phase angle (slight increase in elastic behavior). The addition of 5\% of Plasmix 50/50 leads to a real modification of the binder, with a substantial change of its rheological behavior; the relevant increase in elastic behavior is proved by the decreased phase angles, with a plateau region typical of the formation of a polymer network in the blend [39]. Furthermore, the TTSP is not valid for the blend with $5 \%$ of Plasmix 50/50 as it does not have a unique curve in the Black diagram. This evidence is known for Polymer Modified Bitumen (PMBs) as the Partial Time-Temperature Superposition Principle (PTTSP) [40] since shifting procedures give continuous master curves as the one depicted in Figure 9.

\subsection{Mechanical Characterization of the Dry Mixture}

For evaluating the feasibility of the use of Plasmix as additive in the mixture via dry method, as clarified in the Materials and Methods section, it was considered appropriate to focusing on the resistance to permanent deformation of two dry mixtures: one with the $2 \%$ of "as is" Plasmix, and a second one with the $2 \%$ of Plasmix 50/50 processed by 
extrusion and granulated. The selected dosage in additive (2\%) is typical of this application and production in the laboratory of the dry mixtures was successful, while the $5 \%$ of Plasmix 50/50 was not tested since this amount of waste plastic proved to be excessive during production of the mixture, with negative effect on the surface characteristics of the compacted samples (severe bleeding and excessive surface smoothness).

As can be seen from Figure 11, where the rut depth of the studied mixtures is given as a function of the number of passes, the result of mixture produced with the processed Plasmix 50/50 is not significantly different from those gained by the reference mixture, with no waste plastic. On the other hand, the sample obtained with the Plasmix "as is", provides a significant variation in the rutting resistance at high temperature; the final rut depth at 20,000 passes is about $60.0 \%$ less than the total rut cumulated by the traditional bituminous mixture, with neat bitumen.

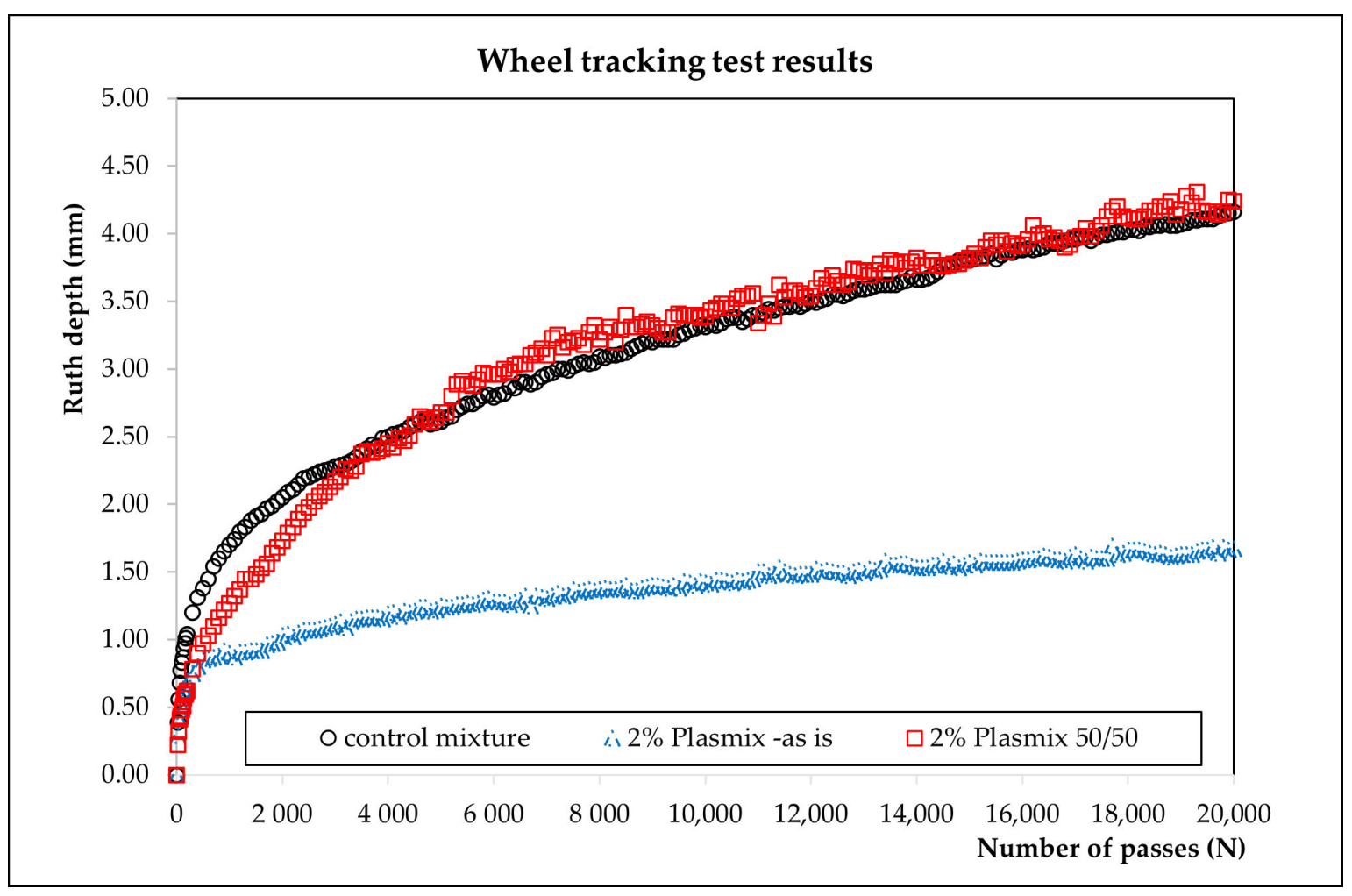

Figure 11. Rut depth of the studied mixtures in the wheel tracking test, as a function of the number of passes.

The abovementioned means that the resistance to permanent deformation is increased by the addition of the Plasmix "as is", via dry method, in the mixture. Comparing the results of the different mixtures subject to rutting test, the "as is" Plasmix performs much better than control one, with no additive, and also than the one with processed Plasmix $50 / 50$ (extruded and granulated); this is probably due to the shredded shape of the material "as is" in thin "flakes" that facilitated its dispersion into the mixture, allowing an adequate interaction between polymer and binder.

Another crucial requirement for the mixtures to be used as wearing courses is water sensitivity. The selected mixture is characterized by a continuous aggregate skeleton that ends up in a packed structure that allows a very low void content, at optimum binder content. Thus, the mixture may be considered as impermeable and the addition of the plastic should enhance this characteristic, thus ending up in a reduced water sensitivity. Therefore, water sensitivity of the dry mixtures will be better investigated in future development of the research, involving mixtures characterized by different void contents. 


\section{Conclusions}

The aim of this work was to investigate the possibility of using very heterogeneous waste plastics as modifiers in bitumen and bituminous mixture, for road application. The study focused on the dry (into the mixture) and wet (into the binder) addition of the Plasmix compound, with the aim of assessing the feasibility of the modification itself.

Based on the experimental results, after appropriate selection of ratio between light and heavy fractions in the compound, accurate choice of the pre-processing operations and related conditions (such as extrusions and granulation, for the wet process) as well as of the content of Plasmix to be added, it is possible to successfully perform both the binder or mixture modifications, also considering the requirements for industrial practice in asphalt plant to be met, in terms of stability of the polymer/bitumen blend as well as in terms of viscosity for pumpability and mixing temperature with aggregate.

For the wet method, where the bitumen is modified with the selected "waste" plastic, the results of the empirical tests as well as those of the rheological tests prove an increase in stiffness and viscosity that is related to an improved resistance to the traffic loads at high temperatures. DSR results also prove a more elastic behavior of the modified binders, compared to that of the neat bitumen. Indeed, chemical characterization of the Plasmix is crucial and further analysis (TGA, etc.) may be useful for defining the most adequate mixing conditions with the bitumen.

For the dry method, where the plastic is added to the hot aggregate right before mixing with the bitumen, a higher resistance to permanent deformation at high temperature is proved. Indeed, further studies are needed, in order to evaluate other crucial requirements for these kinds of applications (wearing courses), related to both mechanical and functional (surface) properties of the mixtures, as well as the overall environmental sustainability of the modification process (both via wet and dry method), in order to account for the overall impacts in terms of air pollutants and global emissions, as related to the production of the modified binders or mixtures.

The results obtained are very promising and suggest further studies to be carried out in order to develop reliable techniques-highly dependent on the base bitumen to be modified as well as on the waste materials to be used-that allow the recycling in road application of large quantities of the Plasmix waste that, otherwise, would be disposed in the landfills.

Author Contributions: Conceptualization, C.C.; methodology, F.P.L.M.; validation, C.C. and R.T.; investigation, C.C.; resources, A.P.; data curation, F.G.; writing-original draft preparation, C.C., R.T. and F.G.; writing - review and editing, C.C.; supervision, project administration and funding acquisition, F.P.L.M. All authors have read and agreed to the published version of the manuscript.

Funding: This research was funded by COREPLA, Italian National Consortium for the Collection and Recycling of Plastic Packages.

Acknowledgments: The authors would like to thank Pierluca Fara for his help in carrying out some laboratory tests.

Conflicts of Interest: The authors declare no conflict of interest.

\section{References}

1. La Mantia, F.P.; Vinci, M. Recycling poly (ethyleneterephthalate). Polym. Degrad. Stab. 1994, 45, 121-125. [CrossRef]

2. Loultcheva, M.K.; Proietto, M.; Jilov, N.; La Mantia, F. Recycling of high density polyethylene containers. Polym. Degrad. Stab. 1997, 57, 77-81. [CrossRef]

3. La Mantia, F.; Curto, D.; Scaffaro, R. Recycling of dry and wet polyamide 6. J. Appl. Polym. Sci. 2002, 86, 1899-1903. [CrossRef]

4. La Mantia, F.P. Recycling of heterogeneous plastics wastes: I-Blends with low-density polyethylene. Polym. Degrad. Stab. 1992, 37, 145-148. [CrossRef]

5. Dintcheva, N.T.; Mantia, F.L. Recycling of the "light fraction" from municipal post-consumer plastics: Effect of adding wood fibers. Polym. Adv. Technol. 1999, 10, 607-614. [CrossRef]

6. Ahmadinia, E.; Zargar, M.; Karim, M.R.; Abdelaziz, M.; Ahmadinia, E. Performance evaluation of utilization of waste Polyethylene Terephthalate (PET) in stone mastic asphalt. Constr. Build. Mater. 2012, 36, 984-989. [CrossRef] 
7. Asare, P.N.A.; Kuranchie, F.A.; Ofosu, E.A. Evaluation of incorporating plastic wastes into asphalt materials for road construction in Ghana. Cogent Environ. Sci. 2019, 5, 1576373. [CrossRef]

8. Rahman, M.T.; Mohajerani, A.; Giustozzi, F. Recycling of waste materials for asphalt concrete and bitumen: A review. Materials 2020, 13, 1495. [CrossRef] [PubMed]

9. Costa, L.M.; Silva, H.M.R.D.; Oliveira, J.R.; Fernandes, S.R. Incorporation of waste plastic in asphalt binders to improve their performance in the pavement. Int. J. Pavement Res. Technol. 2013, 6, 457-464.

10. Zhu, J.; Birgisson, B.; Kringos, N. Polymer modification of bitumen: Advances and challenges. Eur. Polym. J. 2014, 54, 18-38. [CrossRef]

11. Kalantar, Z.N.; Karim, M.R.; Mahrez, A. A review of using waste and virgin polymer in pavement. Constr. Build. Mater. 2012, 33, 55-62. [CrossRef]

12. Nizamuddin, S.; Jamal, M.; Gravina, R.; Giustozzi, F. Recycled plastic as bitumen modifier: The role of recycled linear low-density polyethylene in the modification of physical, chemical and rheological properties of bitumen. J. Clean. Prod. 2020, 266, 121988. [CrossRef]

13. Polacco, G.; Filippi, S.; Merusi, F.; Stastna, G. A review of the fundamentals of polymer-modified asphalts: Asphalt/polymer interactions and principles of compatibility. Adv. Colloid Interface Sci. 2015, 224, 72-112. [CrossRef]

14. Ranieri, M.; Costa, L.; Oliveira, J.R.M.; Silva, H.M.R.D.; Celauro, C. Asphalt surface mixtures with improved performance using waste polymers via dry and wet processes. J. Mater. Civ. Eng. 2017, 29, 04017169. [CrossRef]

15. Saroufim, E.; Celauro, C.; Mistretta, M.C. A simple interpretation of the effect of the polymer type on the properties of PMBs for road paving applications. Constr. Build. Mater. 2018, 158, 114-123. [CrossRef]

16. Celauro, B.; Celauro, C.; Di Francisca, A.; Giuffrè, O. Effect of the construction process on the performances of asphalt paving mixes with crumb rubber from scrap tyres. In Proceedings of the 3rd Eurasphalt and Eurobitume Congress Held, Vienna, Austria, 12-14 May 2004.

17. Celauro, C.; Bosurgi, G.; Sollazzo, G.; Ranieri, M. Laboratory and in-situ tests for estimating improvements in asphalt concrete with the addition of an LDPE and EVA polymeric compound. Constr. Build. Mater. 2019, 196, 714-726. [CrossRef]

18. McNally, T. Polymer Modified Bitumen: Properties and Characterisation; Elsevier: Amsterdam, The Netherlands, 2011.

19. Wu, S.; Montalvo, L. Repurposing Waste Plastics into Cleaner Asphalt Pavement Materials: A Critical Literature Review. J. Clean. Prod. 2020, 280, 124355. [CrossRef]

20. Keijzer, E.E.; Leegwater, G.A.; de Vos-Effting, S.E.; de Wit, M.S. Carbon footprint comparison of innovative techniques in the construction and maintenance of road infrastructure in The Netherlands. Environ. Sci. Policy 2015, 54, 218-225. [CrossRef]

21. Gaestel, C.; Smadja, R.; Lamminan, K. Contribution à la connaissance des propriétés des bitumes routiers. Rev. Gentile Routes Aérodr. 1971, 466, 85-94.

22. Yang, P.; Cong, Q.; Liao, K. Application of Solubility Parameter Theory in Evaluating the Aging Resistance of Paving Asphalts. Pet. Sci. Technol. 2003, 21, 1843-1850. [CrossRef]

23. Read, J.; Whiteoak, D. The Shell Bitumen Handbook, 5th ed.; Thomas Telford Ltd.: London, UK, 2003.

24. Redelius, G. The structure of asphaltenes in bitumen. Road Mater. Pavement Des. 2006, 7, 143-162. [CrossRef]

25. Oliver, J.W.H. Changes in the Chemical Composition of Australian Bitumens. Road Mater. Pavement Des. 2012, 10, 569-586. [CrossRef]

26. Brûlé, B.; Ramond, G.; Such, C. Relationships Among Composition, Structure, and Properties of Road Asphalts. In Developments in Petroleum Science; Elsevier: Amsterdam, The Netherlands, 1994; Volume 40, pp. 427-449.

27. Kriz, P.; Stastna, J.; Zanzotto, L. Glass transition and phase stability in asphalt binders. Road Mater. Pavement Des. 2008, 9 (Suppl. 1), 37-65. [CrossRef]

28. Vetere, M. The Italian Scheme, a Success Story. In Waste Recovery. Strategies, Techniques and Applications in Europe: Strategies, Techniques and Applications in Europe; 17. Italian National Consortium for the Collection and Recycling of Plastic packages COREPLA; FrancoAngeli: Milan, Italy, 2009; p. 224.

29. MIT. Norme Tecniche di Tipo Prestazionale per Capitolati Speciali d'Appalto; Ricerca Realizzata nell'Ambito della Convenzione tra l'Ispettorato per la Circolazione e la Sicurezza Stradale ed il CIRS, MIT-Ministero delle Infrastrutture e dei Trasporti (Italian Ministry for Infrastructures and Transport): Rome, Italy, 2001.

30. Pfeiffer, J.P.; Van Doormaal, P. The rheological properties of asphaltic bitumens. J. Inst. Pet. Technol. 1936, 22, 414-440.

31. Sengoz, B.; Topal, A.; Isikyakar, G. Morphology and image analysis of polymer modified bitumens. Constr. Build. Mater. 2009, 23, 1986-1992. [CrossRef]

32. Celauro, C.; Saroufim, E.; Mistretta, M.C.; La Mantia, F.P. Influence of Short-Term Aging on Mechanical Properties and Morphology of Polymer-Modified Bitumen with Recycled Plastics from Waste Materials. Polymers 2020, 12, 1985. [CrossRef] [PubMed]

33. Williams, M.L.; Landel, R.F.; Ferry, J.D. The temperature dependence of relaxation mechanisms in amorphous polymers and other glass-forming liquids. J. Am. Chem. Soc. 1955, 77, 3701-3707. [CrossRef]

34. Hicks, R.; Cheng, D.; Duffy, T. Evaluation of Terminal Blend Rubberized Asphalt in Paving Applications; Report Number: CP2C-2010102TM; California Pavement Preservation Center: Chico, CA, USA, 2010.

35. Huisingh, D.; Zhang, Z.; Moore, J.C.; Qiao, Q.; Li, Q. Recent advances in carbon emissions reduction: Policies, technologies, monitoring, assessment and modeling. J. Clean. Prod. 2015, 103, 1-12. [CrossRef]

36. Institute, A. The Asphalt Handbook; Asphalt Institute: Lexington, KY, USA, 1962. 
37. Hunter, R.N.; Self, A.; Read, J.; Hobson, E. The Shell Bitumen Handbook; ICE Publishing: London, UK, 2015.

38. Harrigan, E.; Leahy, R.; Youtcheff, J. Superpave Manual of Specifications, Test Methods and Practices; SHRP-A-379. Strategic Highway Research Program; National Academy of Sciences: Washington, DC, USA, 1994.

39. Polacco, G.; Stastna, J.; Vlachovicova, Z.; Biondi, D.; Zanzotto, L. Temporary networks in polymer-modified asphalts. Polym. Eng. Sci. 2004, 44, 2185-2193. [CrossRef]

40. Olard, F.; Di Benedetto, H. General “2S2P1D” model and relation between the linear viscoelastic behaviours of bituminous binders and mixes. Road Mater. Pavement Des. 2003, 4, 185-224. 